\title{
Carbon-Nanotube-Modified Glass Micropipette for Simultaneous Drug Injection and Neural Monitoring
}

\author{
Jung Hwal Shin¹, Geon Hwee Kim¹, Intae Kim¹, Hoon Lim²,+, and Geunbae Lim,3,+
}

\begin{abstract}
Glass micropipettes are widely used for drug injection in neurological studies. To enable these devices to monitor neural activity simultaneously with drug injection, an electrode such as $\mathrm{Ag} / \mathrm{AgCl}$ must be located near or inserted into the glass micropipette to detect electrical signals in vivo. Here, we report carbon-nanotube-modified glass micropipettes (CNGs), which have excellent electrochemical properties such as low impedance and large electrochemical surface area suited for neural recording. In addition, using a standard pressure pump, CNGs can deliver drugs to the target region without bending. Because they are based on standard glass micropipettes, CNGs can readily be applied to traditional equipment, creating opportunities to monitor precisely the drug-injected area.
\end{abstract}

Keywords : Glass micropipette, Carbon nanotubes (CNTs), Carbon-nanotube modified glass micropipettes (CNGs), Impedance, Drug injection

\section{INTRODUCTION}

Glass micropipettes with tip diameters ranging from hundreds of nanometers to a few micrometers are widely used for cell electrophysiology $[1,2]$. Their limitations include brittleness, relatively large size compared to the cell, and the inability to record electrical signals with drug injection simultaneously [3].

Recently, carbon nanotubes (CNTs) of a nanotube geometry have become popular as a material for neural electrodes because of superior electrical properties intrinsic to this geometry [4]. Additionally, CNTs have outstanding biocompatibility and other functional properties [5]. Therefore, microelectrodes have been frequently modified

\footnotetext{
${ }^{1}$ Department of Mechanical Engineering, Pohang University of Science and Technology (POSTECH), Hyoja-dong, Nam-gu, Pohang, Gyungbuk 790-784, Republic of Korea

${ }^{2}$ Department of Emergency Medicine, Soon Chun Hyang University Hospital, 170 Jomaru-ro, Wonmi-gu, Bucheon, Gyenggi-do 420-767, Republic of Korea

${ }^{3}$ School of Interdisciplinary Bioscience and Bioengineering, Pohang University of Science and Technology (POSTECH), Hyoja-dong, Namgu, Pohang, Gyungbuk 790-784, Republic of Korea

${ }^{+}$Corresponding author: Geunbae Lim (limmems@postech.ac.kr) and Hoon Lim (43210@schmc.ac.kr)

(Received : Aug. 14, 2013, Revised : Sep. 5, 2013, Accepted : Sep. 6, 2013)
}

This is an Open Access article distributed under the terms of the Creative Commons Attribution Non-Commercial License(http://creativecommons.org/licenses/bync/3.0)which permits unrestricted non-commercial use, distribution, and reproduction in any medium, provided the original work is properly cited. with CNTs to decrease their impedance and enhance their sensitivity $[6,7]$.

Some groups have introduced carbon nanopipettes (CNPs) to enable electrical measurements while delivering fluids using standard quartz $[8,9]$ and glass micropipettes $[10,11]$. They demonstrated that the CNPs could deliver attoliter volumes of fluids to a precise location; however, such a low flow rate is difficult to apply in intracellular and broad-area injection. In addition, the fabrication of these nanoscale tube-type devices is complex and difficult to reproduce.

The impedance of neural electrodes is an important factor for neural recording because high-impedance electrodes have a lower signal-to-noise ratio (SNR) and thus the neural signals are often lost in the noise. Therefore, the impedance must be lowered to enhance electrode sensitivity, or a larger electric charge must be used for stimulation $[12,13]$. Recently, surface modification technologies using CNTs [6] or conducting polymers [14] have been used to improve the impedance while maintaining the small size of the electrodes.

In this study, we present the successful fabrication of carbon-nanotube-modified glass micropipettes (CNGs), fabricated from standard glass micropipettes. The electrochemical characteristics of the CNGs are compared with those of non-CNT-coated glass micropipettes, and the injection capability of CNGs is determined using fluorescence dye. The results show the superiority of the 
CNGs over non-CNT-coated glass micropipettes, suggesting that the CNGs can record electrical signals concurrently with drug injection.

\section{EXPERIMENTAL}

\subsection{Fabrication of CNGs}

\subsubsection{Dispersion of CNTs}

Single-walled nanotubes (SWNTs) were produced using an arc discharge process with a diameter of 1.0 to $1.2 \mathrm{~nm}$ diameter and a length of 5 to $20 \mu \mathrm{m}$ (Hanhwa Nanotech, Incheon, South Korea). Carboxylate-functionalized SWNTs (SWNT-COOH) were prepared by oxidizing the SWNTs in a strong acid with sonication. First, SWNTs (5 $\mathrm{mg}$ ) were mixed into $50 \mathrm{~mL}$ of concentrated $\mathrm{H}_{2} \mathrm{SO}_{4} / \mathrm{HNO}_{3}$ $(3: 1, \mathrm{v} / \mathrm{v})$ solution and sonicated in an ultrasonication bath for $3 \mathrm{~h}$. Next, the SWNTs suspension was diluted with deionized (DI) water and then centrifuged at $12000 \mathrm{rpm}$ for $10 \mathrm{~min}$. The remaining acid was completely removed by repeated cycles of centrifuging, decanting the supernatant, and washing with DI water, until the $\mathrm{pH}$ of the solution reached 7.4. Finally, the SWNTs were re-dispersed in DI water by ultrasonication.

\subsubsection{Fabrication of CNGs}

Glass capillary tubes with an outer diameter of 1.0 to 1.1 $\mathrm{mm}$, an inner diameter of 0.66 to $0.70 \mathrm{~mm}$, and a length of 4 inch were purchased from Chemglass Life Sciences (USA, CG-1182-01). As illustrated in Fig. 1b, the tubes were pulled by a puller (Narishige, Japan, model PN-30) under the following conditions to obtain a tip of $1 \mu \mathrm{m}$ in diameter. The strength of the main magnet was 118 , the strength of the sub magnet was 14.9, and the temperature of the heater was 101.5. A titanium layer about $0.2 \mu \mathrm{m}$ thick was sputter-coated by sputter-I (SNTEK, Korea, MSS5000) onto the glass micropipette (Fig. 1c). As shown in Fig. 1d, the apex of the glass pipette was then cut by a 30 $\mathrm{kV}$ gallium focused ion beam (FIB, SMI 3050, SII nanotechnology, Inc., Japan). The FIB process was performed by two-step processing. First, a probe current of $90 \mathrm{pA}$ was used to make a rough cut in the glass pipette; the Gaussian ion beam profile causes the end to have a slight slant (Fig. 2b-1). Next, a probe current of $10 \mathrm{pA}$ was applied for 2 to $3 \mathrm{sec}$ to polish the roughly cut area, whose tilt angle was about $2^{\circ} \Delta$, to form a clean and flat surface (Fig. 2b-2). Finally, the titanium-coated glass micropipettes were submerged in a suspension of CNTs to a depth of about $2 \mathrm{~mm}$, and an AC electric field of $20 \mathrm{MHz}$ frequency and 10 Vpp amplitude was applied between them [15]. This caused the CNTs to migrate towards the titaniumcoated glass micropipette by dielectrophoretic (DEP) force; the collected CNTs were compressed by the surface tension of water and physisorbed onto the titanium-coated glass micropipette by Van der Waals forces [5].

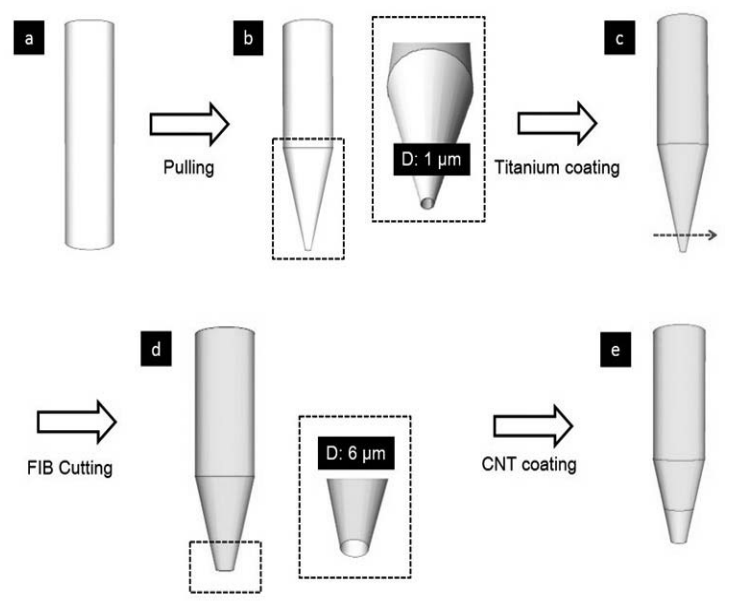

Fig. 1. Schematic of CNG fabrication process; (a) Preparation of glass capillary tube, (b) pulling of glass micropipette, (c) sputter-coating the glass micropipette with titanium. (d) focused ion-beam cutting of the apex of the glass micropipette, and (e) coating the tip apex with CNTs.

\subsection{Mechanical properties of titanium-coated glass micropipettes}

The mechanical properties of titanium-coated glass micropipettes and bare glass micropipettes were tested by slowly inserting the pipettes $4 \mathrm{~mm}$ from the surface of a soft soybean curd, using a micromanipulator, at an insertion rate of about $40 \mu \mathrm{m} / \mathrm{s}$.

\subsection{Electrochemical measurements of CNGs}

\subsubsection{Impedance measurements}

The impedance of the CNGs was measured using an impedance analyzer (HP 4192A, Hewlett-Packard, Palo Alto, CA, USA) within the frequency range of $100 \mathrm{~Hz}$ to $10 \mathrm{kHz}$, using a $\mathrm{CNG}$ as the working electrode, $\mathrm{Ag} / \mathrm{AgCl}$ 
as the reference, and $\mathrm{Pt}$ as the counter electrode in phosphate-buffered saline (PBS pH 7.4).

\subsubsection{Cyclic voltammetry (CV) measurements}

The CV response of CNGs was measured in in a solution of $0.02 \mathrm{M} \mathrm{K}_{3} \mathrm{Fe}(\mathrm{CN})_{6}$ and $0.5 \mathrm{M} \mathrm{KCl}$ using a three-electrode configuration (Modulab System, Solartron Instruments, Elmsford, NY, USA). The three-electrode cell was composed of an $\mathrm{Ag} / \mathrm{AgCl}$ reference electrode, a $\mathrm{Pt}$ counter electrode, and a CNG test electrode. The potential was varied from -0.2 and $1.2 \mathrm{~V}$ at a scan rate of $100 \mathrm{mV} / \mathrm{s}$ beginning at the open-circuit potential and sweeping in the positive direction. Prior to recording data, several cycles were swept to guarantee that the electrode had reached a stable state.

\subsection{Fluorescence dye injection using CNGs}

A fluorescent dye was purchased from Invitrogen (Alexa 488 carboxylic acid, succinimidyl ester, mixed isomers, catalog number a20000). The CNGs were filled with a fluorescent dye, following which a syringe pump was used to inject the dye into a bowl containing DI water. The flow rate was varied from 5 to $20 \mu \mathrm{L} / \mathrm{min}$, and fluorescence images were obtained using a fluorescence microscope (Olympus IX71 inverted microscope at 40×magnification).

\section{RESULTS AND DISCUSSION}

\subsection{Fabrication of CNGs}

Glass micropipettes are widely used in drug injection, but because of their insulating properties, they cannot record outer electrical signals simultaneously with injection. To address this limitation, titanium was coated onto glass micropipettes.

Figure 1a-e illustrates the CNG fabrication process. First, glass capillary tubes of approximately $1 \mu \mathrm{m}$ diameter were pulled by a puller, and had a tip diameter of approximately $1 \mu \mathrm{m}$. To confer conductive characteristics, the pulled glass micropipettes were coated with titanium, and the deposited thickness was about 2,000 (Fig. 2a). The tip diameter after cutting by FIB was about $6 \mu \mathrm{m}$ (Fig. 2b) which is the diameter most generally adopted for drug injection in vivo. To decrease the impedance of the titanium-coated glass micropipette, CNTs were deposited using an AC electric field (Fig. 2d). In our previous research, CNT-based neural electrodes (CNEs) were made using same synthesis conditions as the CNGs [16]. The non-covalent attachment of CNTs onto the micropipette surface provides sufficient mechanical stability in the brain.
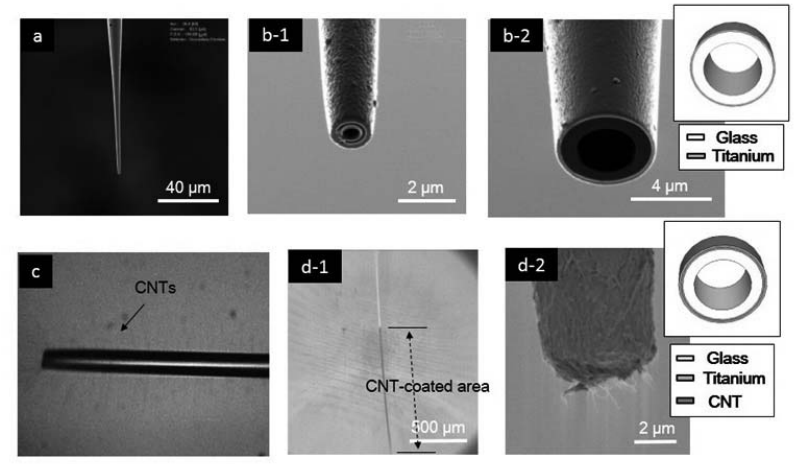

Fig. 2. Scanning electron microscopy images of CNG fabrication process; (a) SEM image of titanium-modified glass micropipette, (b) SEM image after focused ion beam cutting at the end of the glass micropipette, (c) optical microscopy image during CNT coating, and (d) SEM image after CNT coating (CNG).

\subsection{Mechanical characterization of titanium-coated glass micropipettes}

The rigidity of implanted electrodes is important in locating the desired position. For example, a very flexible electrode might not be able to locate the desired position, and this can decrease the experiment reliability. Soft soybean curd was used to test because its mechanical properties are most similar to those of the brain.
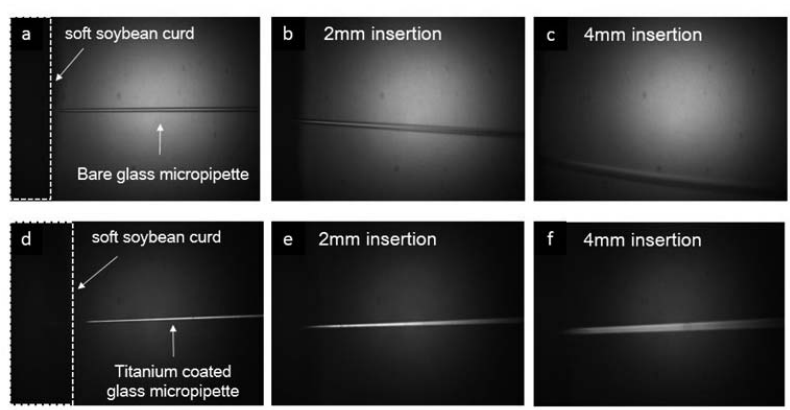

Fig. 3. Optical microscopy images during testing of the mechanical properties; (a) Before insertion of the bare glass micropipette, (b) after $2 \mathrm{~mm}$ insertion, (c) after $4 \mathrm{~mm}$ insertion, (d) before insertion of a CNG, (e) after $2 \mathrm{~mm}$ insertion, and (f) after $4 \mathrm{~mm}$ insertion. 
The titanium-coated glass micropipettes were carefully inserted into a soft soybean curd using a micromanipulator, and their shape was observed via optical microscopy. Figure 3a-c shows optical images of a bare glass micropipette. During the insertion, the bare glass micropipette gradually became bent. Glass capillary tubes are typically quite brittle, but after pulling, they become too flexible for neural injections. Figure $3 \mathrm{~d}-\mathrm{f}$ shows the shape of a titanium-coated glass micropipette. During insertion into the soybean curd, the titanium-coated glass micropipette maintained its original shape. These results demonstrate that the titanium coating can grant sufficient rigidity to the glass micropipettes to allow their use in brain transplant .

\subsection{Electrochemical characterization of CNGs}

The impedance is important for neural recording because the lower the impedance, the higher the sensitivity. CNTs can be used for decreasing the electrode impedance because of their high electrochemical surface area (ESA)/geometric surface area (GSA) ratio [4]. Because the charge transfer mechanism of the CNTs is very similar to that of PEDOT, CNTs can work as ion-to-electron transducers for neural recording [17].

The blue dots in Figure 4a correspond to the impedance of non-CNT-coated electrodes (titanium-coated glass micropipette) while that of CNG in PBS solution is shown using red dots. The impedance of CNG was decreased compared with that of non-CNT-coated electrodes at all frequencies $(0.1$ to $10 \mathrm{kHz})$. Figure $4 \mathrm{~b}$ shows each electrode's impedance before and after CNT coating, and the average impedance of the two electrodes at the biologically relevant frequency of $1 \mathrm{kHz}$. The average impedance of non-CNT-coated electrodes and CNGs was 9.3 and $6.8 \mathrm{k} \Omega$, respectively.

Figure $4 \mathrm{c}$ shows the CV response of non-CNT-coated electrodes (blue dots) and CNGs (red dots). The difference of the currents $(\Delta i)$ between positive and negative potential cycles corresponds to the sum of the charging and discharging currents at the electrode-electrolyte interface. The specific capacitance $\left(\mathrm{C}_{0}\right)$ relative to the electrochemical surface is described in Equation. 1.

$$
\mathrm{C}_{0}=\Delta \text { i/ } 2 \mathrm{~V}
$$

( $\Delta i$ : average current, $\mathrm{v}$ : the potential sweep rate)
The specific capacitance of the CNG is about 100 times larger than that of non-CNT-coated electrode. The significant increase in specific capacitance comes from the enhanced faradaic reaction of the CNT during the potential cycling. In conclusion, the enhanced faradaic reaction of the CNT increases the specific capacitance and decreases the impedance of the CNGs relative to non-CNT-coated electrodes. This method to decrease the impedance of CNGs could improve their sensitivity for neural recording.
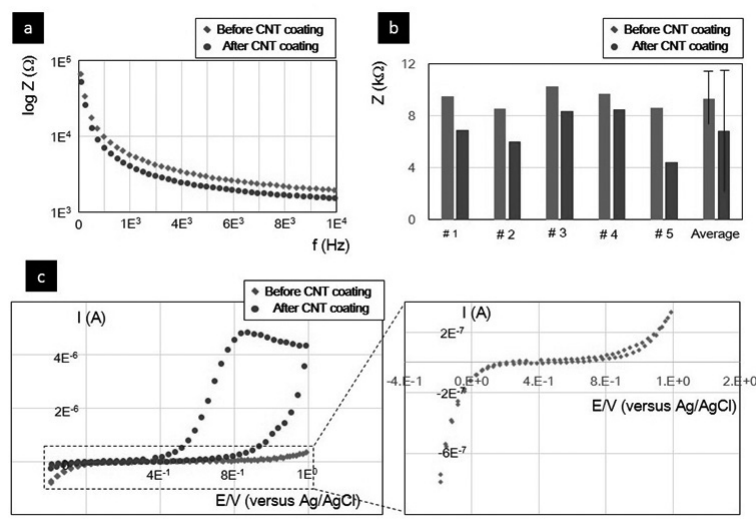

Fig. 4. Electrochemical properties of the CNGs; (a) The impedance of CNG and non-CNT-coated electrodes at 0.1 to $10 \mathrm{kHz}$, (b) the impedances of CNGs and non-CNTcoated electrode at $1 \mathrm{kHz}(\mathrm{n}=5)$, and (c) cyclic voltammogram of $\mathrm{CNG}$ and non-CNT coated electrodes in a solution of $20 \mathrm{mM} \mathrm{K}_{3} \mathrm{Fe}(\mathrm{CN})_{6}$ and $500 \mathrm{mM} \mathrm{KCl}$ (scan rate: $100 \mathrm{mV} \mathrm{s}^{-1}$ )

\subsection{Use of CNGs in drug injection}

Because the CNGs are based on commercially available glass capillary tubes, they can be easily adapted to standard
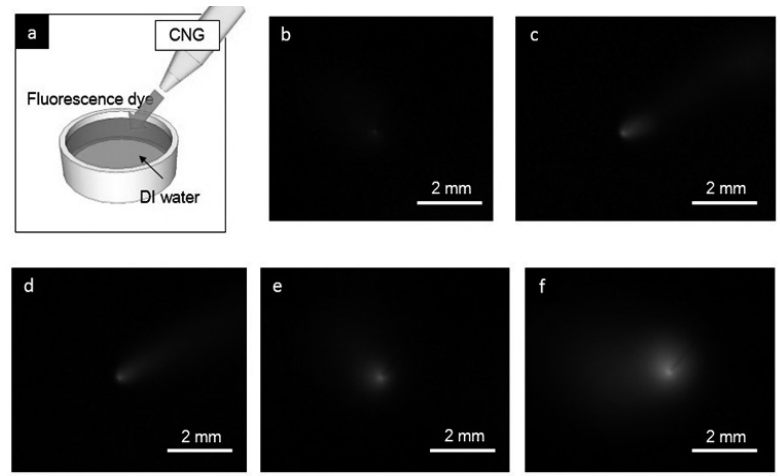

Fig. 5. Drug injection test of CNG; (a) Schematic of drug injection experiment, (b) before injection, (c) flow rate: $5 \mu \mathrm{L} / \mathrm{min}$, (d) flow rate: $10 \mu \mathrm{L} / \mathrm{min}$, (e) flow rate: $15 \mu \mathrm{L} / \mathrm{min}$, and (f) flow rate: $20 \mu \mathrm{L} / \mathrm{min}$. 
cell physiology equipment. Injection experiments of CNGs were conducted using the green fluorescent dye Alexa 488 having (Fig. 5a). The injection flow rates, ranging from 5 to $20 \mu \mathrm{L} / \mathrm{min}$, were generated using a syringe pump. Figure $5 \mathrm{~b}$ shows that before injection, a small amount of dye leaks out by diffusion. Figure $5 \mathrm{c}-\mathrm{f}$ shows that the green fluorescence gradually increases with increasing flow rate. These results demonstrate that the CNGs can be easily used in drug injection using a standard syringe pump.

\section{CONCLUSIONS}

In summary, carbon-nanotube-modified glass micropipettes (CNGs) were developed as drug injectable electrodes. Coating glass micropipettes with titanium rendered them conductive, to detect electrical signals, and the titanium coating also granted sufficient rigidity for brain implantation. CNT coating decreased the impedance of CNGs, significantly increasing the specific capacitance and enhancing the faradaic reaction of CNT. Furthermore, the CNGs can easily deliver drugs using a standard syringe pump. However, the impedance of the present CNGs is too low for their use as neural electrodes, because of their low selectivity. In future work, we envision insulating the side of the $\mathrm{CNG}$ for high selectivity and measuring neural signals simultaneously with drug injection into living mouse brains.

\section{ACKNOWLEDGMENT}

This work was supported by the National Research Foundation of Korea (NRF) grant funded by the Korea government (MEST) (No. 2012R1A2A2A06047424) and the National Research Foundation of Korea (NRF) grant funded by the Korea government (MEST) (No.2012R1A1A2007580).

\section{REFERENCES}

[1] T. V. Tsulaia, N. L. Prokopishyn, A. Yao, N. D. V. Carsrud, M. C. Carou, D. B. Brown, B. R. Davis, and J. Y. Brown, "Glass needle-mediated microinjection of macromolecules and transgenes into primary human mesenchymal stem cells”, J. Biomed. Sci., Vol. 10, pp. 328-336, 2003.
[2] H. Matsuoka and M. Saito, "High throughput microinjection technology toward single-cell bioelectrochemistry", Electrochemistry, Vol. 74, No. 1, pp. 12-18, 2006.

[3] D. J. Stephens and R. Pepperkok, Stephens DJ and Pepperkok R., "The many ways to cross the plasma membrane", Proc. Natl. Acad. Sci. U. S. A., Vol. 98, No. 8, pp. 4295-4298, 2001.

[4] S. F. Cogan, "Neural stimulation and recording electrodes", Annu. Rev. Biomed. Eng., Vol. 10, pp. $275-$ 309, 2008.

[5] T. An, K. S. Kim, S. K. Hahn, and G. Lim, "Real-time, step-wise, electrical detection of protein molecules using dielectrophoretically aligned SWNT-film FET aptasensors", Lab Chip, Vol. 10, No. 16, pp. $2052-$ 2056, 2010.

[6] E. W. Keefer, B. R. Bolterman, M. I. Romero, A. F. Rossi, and G. W. Gross, "Carbon nanotube coating improves neuronal recordings", Nat. Nanotechnol., Vol. 3, No. 7, pp. 434-439, 2008.

[7] S. Minnikanti and N. Peixoto, "Implantable electrodes with carbon nanotube coatings", In: Marulanda JM, editor. Carbon Nanotub. Appl. Electron Devices, InTech, 2011.

[8] Schrlau MG, Falls EM, Ziober BL, and Bau HH, "Carbon nanopipettes for cell probes and intracellular injection”, Nanotechnology, Vol. 19, No. 1, pp. 015101-015105, 2008.

M. G. Schrlau, E. M. Falls, B. L. Ziober, and H. H. Bau,

[9] M. G. Schrlau, N. J. Dun, and H. H. Bau, "Cell electrophysiology with carbon nanopipettes", ACS Nano, Vol. 3, No. 3, pp. 563-568, 2009.

[10] R. Singhal, S. Bhattacharyya, Z. Orynbayeva, E. Vitol, G. Friedman, and Y. Gogotsi, "Small diameter carbon nanopipettes", Nanotechnology, Vol. 21, No. 1, p. 015304, 2010.

[11] R. Singhal, Z. Orynbayeva, R. V. Kalyana Sundaram, J. J. Niu, S. Bhattacharyya, E. A. Vitol, M. G. Schrlau, E. S. Papazagiou, G. Friedman, and Y. Gogotsi, "Multifunctional carbon-nanotube cellular endoscopes", Nat. Nanotechnol., Vol. 6, No. 1, pp. 57-64, 2011.

[12] G. E. Loeb, R. A. Peck, and J. Martyniuk, "Toward the ultimate metal microelectrode", J. Neurosci. Methods, Vol. 63, pp.175-183, 1995.

[13] D. A. Robinson, "The electrical properties of metal microelectrodes", Proc. IEEE, Vol. 56, pp. $1065-$ 1071, 1968. 
[14] K. A. Ludwig, J. D. Uram, J. Yang, D. C. Martin, and D. R. Kipke, "Chronic neural recordings using silicon microelectrode arrays electrochemically deposited with a poly(3,4-ethylenedioxythiophene) (PEDOT) film", J. Neural Eng., Vol. 3, pp. 59-70, 2006.

[15] T. An, W. Choi, E. Lee, I. Kim, W. Moon, and G. Lim, "Fabrication of functional micro- and nanoneedle electrodes using a carbon nanotube template and electrodeposition", Nanoscale Res. Lett., Vol. 6, p. 306, 2011

[16] J. H. Shin, G. B. Kim, E. J. Lee, T. An, K. Shin, S. E.
Lee, W. Choi, S. Lee, C. Latchoumane, S. H. Shin, and G. Lim, "Carbon-nanotube-modified electrodes for highly efficient acute neural recording”, $A d v$. Healthc. Mater., 2013.

[17] G. A. Crespo, S. Macho, J. Bobacka, F. X. Rius, "Transduction mechanism of carbon nanotubes in solid-contact ion-selective electrodes", Anal. Chem., Vol. 81, No. 2, pp. 676-681, 2009.

[18] Conway BE, Electrochemical Supercapacitors: Scientific Fundamentals and Technological Applications, Springer, 1999. B. E. Conway, 